npr. na str. 91, 99, 100, 279), da jeziki sveta še niso dovolj dobro opisani (tudi na sinhroni ravnini, ne le na diahroni), da tudi najbolj pogosto omenjane slovnične kategorije (subjekt) in pojavi (jezikovna sprememba) niso dovolj jasni, da bi bili zares uporabni za tipološke raziskave, predpostavljene tipološke značilnosti jezikov, ki služijo kot osnova za nadaljnje razmišljanje, pa so same po sebi sporne. Kljub temu Matasović na zgornje vprašanje odgovarja pritrdilno: značilnosti, ki jih mora izpolnjevati neka beseda, da jo lahko imenujemo subjekt, ni potrebno vnaprej natančno opredeliti, pojmu lahko sproti dodajamo nove morfosintaktične poteze, če jih le primerno pojasnimo $\mathrm{v}$ odnosu do iste kategorije $\mathrm{v}$ drugih jezikih, nevarnostim, da bi kot enake primerjali jezikovne spremembe, ki kažejo le navidezno podobnost začetne in končne stopnje, se izognemo z opazovanjem vmesnih stopenj razvoja in točnim definiranjem kategorij, tipoloških študij pa se lahko lotevamo, ker od nadaljnjega opisovanja jezikov ni pričakovati radikalno novih ugotovitev o slovničnih značilnostih jezikov. Relevantnost ugotovitev ob takih izhodišcih seveda postaja vprašljiva, izkušnje pa nas učijo, da se celo $\mathrm{v}$ najbolje raziskanih jezikih neprestano pojavljajo nove, povsem drugačne interpretacije izpričanih jezikovnih dejstev. To pa so že vprašanja, ki presegajo okvir učbenika in jih bo jezikoslovna znanost morala reševati $v$ drugačnem kontekstu.

Marina Zorman

\title{
Jacqueline Picoche, Jean-Claude Rolland, Dictionnaire du français usuel. 15000 mots utiles en 442 articles. Bruxelles: De Boeck/Duculot. 2002 (Deux éditions dispo- nibles: édition papier et édition électronique sur cédérom PC/Mac).
}

Le Dictionnaire du français usuel ( $D F U$ ) est le résultat impressionnant d'une tentative originale qui sort du cadre habituel de ce qu'on s'est accoutumé à appeler «dictionnaires d'apprentissage». Avant d'entamer un rapide examen lexicographique et dictionnairique proprement dit, il convient de mentionner l'initiative méritoire des éditeurs de décliner le produit en deux versions, l'une sur papier et l'autre sur cédérom.

Le dictionnaire est l'aboutissement de recherches systématiques dans le domaine de la lexicologie et de la didactique du vocabulaire français que Jacqueline Picoche (aidée par ses collaborateurs) mène depuis de nombreuses années. Sa théorie, qui présidait aussi à l'élaboration du présent dictionnaire, s'articule essentiellement autour de trois axes méthodologiques: les études statistiques sur la fréquence des mots, la psychomécanique de Gustave Guillaume (centrée sur les notions de «subduction», de "cinétisme» et de «signifié de puissance») et la méthode des champs actanciels inspirée librement des travaux de Lucien Tesnière. 
Le dictionnaire est conçu comme un outil d'apprentissage destiné en premier lieu, et à la différence des dictionnaires d'apprentissage «classiques», aux enseignants de français langue maternelle ou étrangère. Il vise à dresser un panorama cohérent du lexique qui permettrait un enseignement systématique d'un large vocabulaire usuel. Le péritexte du dictionnaire («Présentation et mode d'emploi») propose en outre un certain nombre d'exercices de vocabulaire (pp. 18-20), d'ailleurs plus amplement développés dans la Didactique du vocabulaire français (Nathan, 1993) du même auteur. Il va sans dire que ce dictionnaire peut profiter aussi aux apprenants allophones de niveaux avancés.

Le $D F U$, aux dires des rédacteurs, n'est pas destiné à la consultation. Pour illustrer qu'il ne s'agit vraiment pas d'un dictionnaire de «dépannage» (le terme est emprunté à Robert Galisson), on n'a qu'à jeter un coup d'œil sur la nomenclature qui est limitée à 15000 unités lexicales, ce qui parait insuffisant pour l'usage réceptif. Comme nous le verrons par la suite, la macrostructure et la microstructure combinant le dispositif abécédaire (donc sémasiologique) et le dispositif sémantique (onomasiologique) ne facilitent nullement l'accès aux informations pour un usager à la recherche d'une information ponctuelle.

Le $D F U$ recense, outre les unités lexicales, les éléments de formation d'origine latine ou grecque: par exemple, l'entrée doigt est suivie de deux bases, digit- et dactyl-, l'entrée force de dynam-, l'entrée enfant de puer- et péd(o)-, etc. En revanche, les rédacteurs ont écarté les mots outils (articles, pronoms, conjonctions, la plupart des prépositions).

La nomenclature du dictionnaire s'agence en 442 articles. Les entrées des articles sont constituées des mots de haute fréquence (au nombre de 613) qui, selon les auteurs, présentent «le noyau dur» du lexique français. Pour en établir le répertoire, les rédacteurs se sont fondés sur plusieurs listes de fréquences existantes ainsi que sur les travaux statistiques d'Étienne Brunet. Les entrées regroupent parfois jusqu'à deux ou trois mots qui entretiennent différents types de relations sémantiques (antonymie: matin et soir, lourd et léger, construire et détruire...; parasynonymie: opinion et avis, savoir et connaître...; dérivation sémantique: tomber et chute) ou morphosémantiques (mourir et mort, valoir et valeur...). Un tel traitement permet aux utilisateurs allophones de mieux pénétrer dans la structure lexicale du français: l'utilisateur a la possibilité, par exemple, de mettre à profit la description parallèle de savoir et connaître, puisqu'il est nécessaire de bien saisir les différences sémantiques entre ces deux mots, afin d'apprendre leurs sens respectifs. Malheureusement, dans la version papier, les non premiers éléments des entrées ne sont pas faciles à trouver (l'index en fin d'ouvrage ne reprend pas les mots figurant en entrées): ainsi, un utilisateur qui chercherait le verbe plaire ne le trouvera pas directement sous cette entrée étant donné que le mot est regroupé avec aimer sous la lettre A. Comment l'utilisateur pourrait-il savoir que plaire est regroupé précisément avec aimer et non pas, par exemple, avec plaisir sous $\mathrm{P}$ ou avec ennui sous $\mathrm{E}$ ? 
Les autres mots de la nomenclature n'apparaissent qu'au niveau microstructurel: ils constituent de vastes réseaux lexicaux ou «champs actantiels» ayant des «mots-entrées» (ou mots-vedettes) comme points de départ. Deux types d'articles sont à distinguer:

- l'entrée est un verbe, un adjectif ou un nom abstrait: l'article spécifie les actants et leurs traits de sélection (par exemple, humain, concret...) - chacune des divisions de sens est introduite par une phrase-exemple (Jean a donné une montre à Jeannot, s.v. donner), qui est suivie d'un schéma abstrait (Al humain, donne $A 2$ concret à A3 humain, s.v. donner). Après une définition succincte, l'article inventorie les éléments des champs actanciels (à savoir, les dénominations des actants spécifiques, leurs qualifications, les dérivés sémantiques ou morphologiques des mots-vedettes) et introduit des (para)synonymes et des antonymes.

- l'entrée est un nom concret: l'article donne les verbes typiques dont le nom en question peut être actant (par exemple, s.v. feu: Jean allume un feu de bois, le feu brûle, le feu baisse et s'éteint...), il donne ses substituts, ses qualifications usuelles, ses compléments, etc. Ce type d'article privilégie les relations paradigmatiques et tend vers l'encyclopédisme.

Pour faciliter la consultation, les mots constitutifs des champs actanciels sont présentés en petites capitales maigres ou grasses et récapitulés dans l'index final qui, pour chaque mot, fournit des renvois à l'article ou aux articles où le mot en question apparait.

La méthode des champs actanciels a mené les rédacteurs à inclure un certain nombre de mots qui, étant archaïques, littéraires ou trop spécialisés, ne peuvent que très difficillement être considérés comme des mots fréquents ou usuels. Citons, à titre d'exemple: bliaut, robin, rigoriste, ratiociner, orpailleur, madré, corselet, contrit, contrition, chiourme.

Le $D F U$ ne doit pourtant pas être pris pour un dictionnaire onomasiologique traditionnel, c'est-à-dire, pour un dictionnaire analogique ou un thésaurus. Les mots qui constituent les entrées du $D F U$ sont, dans la plupart des cas, des mots (souvent, il s'agit de verbes) de haut degré de polysémie, de sens général, abstrait et plutôt vague. Les dictionnaires onomasiologiques, au contraire, optent pour des entrées nominales et des mots dont la valeur informative est plus élevée. Pour établir leurs microstructures, ces derniers s'appuient principalement sur des relations de nature conceptuelle, tandis que le $D F U$ privilégie des relations linguistiques, lexicales, qu'elles soient syntagmatiques ou paradigmatiques.

Il est très important que le $D F U$ en tant que dictionnaire visant des allophones offre à ses utilisateurs la possibilité d'accéder aux connotations culturelles que véhiculent les mots: dans l'esprit d'un français, le muguet, par exemple, est inséparable de l'idée $\mathrm{du} 1^{\mathrm{er}}$ mai, l'accordéon évoque le bal musette, les dragées rappellent un baptême, une communion ou un mariage.

Une attention particulière est accordée au traitement de tous les types d'expressions figées: aux phrasèmes (à contre cœur, jouer gros jeux, faire une toilette de chat, 
malade comme un chien...), aux collocations (contracter une dette, relever un défi, joueur invétéré, mûrement réfléchi) et aux pragmatèmes (Je vous en prie!, Pensezvous!, Tu penses!, Pendant que j'y pense!). Ces expressions qui apparaissent en italique, comme d'ailleurs toutes les séquences non métalinguistiques (exemples, constructions syntaxiques typiques), sont, de plus, signalées par un signe particulier (ף) qui avertit l'usager que «[c]e qui suit est figé» ( $D F U$, p. 23$)$. Ce moyen ne semble pas susciter de difficultés dans la mesure où ces expressions figées sont neutralisées ou insérées dans des séquences métalinguistiques (en romain, dans le dictionnaire):

«Pour un cheval, ๆ marcher au pas est l'allure la plus lente, qui s'oppose au TROT et au GALOP» (s.v. marcher et pas)

Il en va différemment pour les expressions qui font partie de phrases complètes: le signe đ ne marquant que les débuts des expressions, on peut se demander à juste titre si de nombreux allophones, malgré une bonne maîtrise du français, n'hésiteront pas sur les limites finales de ces expressions:

«Luc $\lceil$ fait ses premiers pas dans la carrière de comptable: il débute. - Le professer explique I pas à pas un problème complexe: il l'analyse et expose une à une chaque donnée.» (s.v. marcher et pas)

Dans la première séquence, l'expression figée se limite-t-elle à faire les premiers pas? Quel est le rôle de la préposition dans? Peut-être que faire les premiers pas ne peut être suivi que de carrière? Aucun moyen typographique ne nous permet de trancher.

Signalons que, à notre connaissance, le DFU est le seul dictionnaire, à l'exception remarquable des deux volumes parus de la dernière $\left(9^{e}\right)$ édition du Dictionnaire de l'Académie française, qui applique systématiquement les Rectifications et recommandations orthographiques, proposées par le Conseil supérieur de la langue française, approuvées par l'Académie française et publiées dans le Journal officiel, le 6 décembre 1990.

Pour conclure ce rapide parcours, abordons encore très brièvement la version électronique. Il convient de constater que le cédérom, $d$ 'installation facile sur le disque dur, s'en tient à la seule reprise de la version papier, mais facilite, toutefois, considérablement l'accès aux informations ainsi-que leur consultation. Le cédérom dispose d'un moteur de recherche très performant (recherche alphabétique, recherche simple, recherche d'un mot sous toutes ses formes fléchies, recherche en plein texte, recherche avec une tolérance d'erreurs dans la graphie, recherche à l'aide des jokers et des opérateurs logiques...), il permet la navigation hypertexte, l'affichage simultané de deux articles, le changement de la taille des caractères; il est pourvu de toutes les fonctions bureautiques essentielles (imprimer, copier/coller et exporter vers un logiciel de traitement de texte). II n'est pas surprenant non plus que le cédérom d'un dictionnaire envisagé 
comme outil d'apprentissage offre aux utilisateurs la possibilité de créer leur propre sélection d'informations intitulée «Mon dictionnaire». On aurait cependant souhaité que quelques fonctionnalités additionnelles soient fournies dans la version électronique - mentionnons-en deux: la sonorisation des mots et des expressions idiomatiques susceptibles de soulever des difficultés de prononciation et l'indication systématique et facilement accessible d'une information morphologique complète pour un mot donné (la conjugaison pour un verbe, le féminin et le pluriel pour un nom ou un adjectif).

Gregor Perko

Oana Sălişteanu Cristea, Introduzione alla dialettologia italiana, Tra lingua e dialetto, Editura DAIM, Bucureşti 2002, 160 pagine

1. La professoressa dall'Ateneo di Bucarest, in cui insegna lessicologia, filologia e dialettologia italiana, ci ha regalato due anni fa un primo volume dedicato all'italiano, Prestito latino - elemento ereditario nel lessico della lingua italiana. Doppioni e varianti, Praga 2000. Adesso la studiosa ci offre un'altra opera, citata nel titolo della nostra recensione. Il libro, diciamolo subito, trascende i limiti della filologia italiana, essendo di notevole interesse e importanza per varie altre discipline linguistiche.

2. L'articolazione del volume è la seguente (tra parentesi le pagine): Indice (3-4), Premessa (5-6), Tabella dei simboli dell'API (7-8), I. Lingua e dialetto in Italia (9-33), II. Il repertorio linguistico degli Italiani (34-55), III. Varietà substandard della lingua italiana (56-110), IV. Breve descrizione delle principali varietà regionali (111-142), Appendici (143-148), Bibliografia (oltre 170 titoli; 146-157).

3. La Premessa annuncia in sintesi le linee direttrici dell'esposizione della materia: un rapido cenno dell'interesse dell'Autrice per la "selva dei dialetti italiani", i nuovi indirizzi della linguistica italiana, dall'interesse tradizionale per le isoglosse al processo di italianizzazione delle parlate locali e al substandard (con l'approccio sociolinguistico al primo piano). Il centro del lavoro è pertanto dato dalle varietà substandard dell'italiano. Alla breve descrizione del contenuto seguono le informazioni sulle fonti (con abbondanti citazioni dei più autorevoli studiosi) e sull'API, per terminare con il ringraziamento al professore Marius Sala, agli altri studiosi e a tutti coloro che in qualsiasi senso hanno aiutato l'Autrice.

4. Il primo capitolo traccia l'evoluzione storica e lo spostamento dell'interesse degli studi linguistici in Italia: non più purismo dedicato alla lingua letteraria bensì l'oralità. In seguito: origine, significato e discussione del concetto e del termine dialet- 